\title{
Hospital 5.0: Enhancing Nurses with the Use of Wearables
}

\author{
Stéphanie Gauttier \\ University of Twente \\ Drienerlolaan 5, 7522NB Enschede \\ s.e.j.gauttier@utwente.nl
}

\begin{abstract}
The idea of enhancing humans physically, cognitively, and psychologically is promoted by transhumanists. It requires from technology to focus on one's individual abilities so as to improve them beyond what is possible at the natural state. While enhancement was long the realm of cyborgs and referred to a lonely use of technology, this concept could also be applied to organisations. This paper proposes an analysis of the concept of 'enhancement' applied to the workforce and demonstrates how one could design an intervention to enhance nursing staff in hospitals. Dimensions to consider when developing technology for enhancement in the workplace are identified.
\end{abstract}

Human enhancement, Social Embeddedness, IS in healthcare, Wearables, Ethics

\section{INTRODUCTION}

Technology is being increasingly used to enhance humans, i.e. amplify or extend their physical, cognitive, psychological abilities beyond what is naturally possible at the natural state, transforming humans. Such a vision is promoted by transhumanists and cyborgs, who focus on the individual and personal character of enhancement. At the same time, companies have started to provide microchip implants to employees for the sake of convenience. They also use artificial intelligence to augment their decision-making processes. Thinking of how enhancement can be engineered in the workplace is thinking about the next revolution that can happen at work. Amongst workplaces that could benefit from such a change, we can find hospitals. The hospital of the future is defined by digitalization at all levels: for operational purposes and for patients (Hospital 4.0), but also for the staff (Hospital 5.0). This paper looks at how technology can be used to enhance nurses.

The academic discourse on enhancement in the workplace is scarce and exploring the potential impact of enhancement onto management (Gauttier, 2017). This paper presents dimensions to take into account to study and design technologies to enhance nurses. Firstly, a conceptual analysis is proposed. Secondly, we look at what abilities nurses would like to have enhanced through technology. Thirdly, we present the choice of technology to achieve this form of enhancement, focusing on wearable technology as such technology is available and presents its own acceptance and ethical considerations. Fourthly, we discuss the elements that need to be taken into account to design the use of such technology to be enhancing. The relevance of these elements proposed can be tested in future research.

\section{CONCEPTUAL ANALYSIS OF ENHANCEMENT}

\subsection{Enhancement, a concept initially applied to individuals}

Human enhancement aims at the transformation of individual's abilities in order to make them more performant than at the natural state, so they can reach a state of happiness and well-being (More, 2013a). Enhancement is linked to personal abilities, and as such one can expect that the enhancement technology is adaptable or tailored made to fit individuals. Enhancement can be temporary or permanent (Wainwright, 2015). It is accepted as the right of an individual to experiment on one's body.

This 'experimentation' can take three forms: physical, cognitive, and psychological (More, 2013a). Physical enhancement touches to new abilities of the body, new limbs, or a way to be more performant than at the natural state. Cognitive enhancement is linked to the amplification or extension of one or several cognitive processes such as attention, memory, understanding (Xia and Maes, 2013; Bostrom and Sandberg, 2009) and hierarchies (Engelbart, 1962), or a relief of cognitive load allowing for better performance. It is sometimes seen as intelligence augmentation (Engelbart, 1962), i.e. leading to better, faster, and new ways to 
solve problems. Psychological enhancement is linked to an amplification or extension of mental wellbeing and emotional intelligence skills (satisfaction, lack of stress, empathy). These forms of enhancement are linked: physical enhancement can be linked to cognitive enhancement as the senses coming from the body or the energy saved by the body allows to process elements in a new, more efficient manner. Psychological enhancement might relieve signs of physical stress and might allow for better cognitive skills such as attention or memory. Physical, cognitive and psychological enhancement is meant to lead to moral enhancement (Anderson, 2012). To achieve either of these forms of enhancement, one can target moods, abilities, and performance (Baertschi, 2011).

Enhancement can be achieved through different means: pharmaceutical, neurological, technological. When intelligence augmentation is concerned, Engelbart suggests artefacts, methodologies, training. Enhancement technologies are different from technologies usually used at work in so far as they 1) are used on a (quasi)-continuous basis, while tools are used punctually; 2) aim at the improvement of intrinsic abilities vs the pursuit of extrinsic tasks; 3) they can be used without the express intent of the user, while tools are consciously employed. They can be seen as similar to learning technologies, as training is also considered a way to enhancement (Engelbart, 1962). The difference is that enhancement technologies are assessed against natural abilities, while learning is assessed against prior knowledge. Learning is based on standards of development and for all, while enhancement transforms what is seen as standard and aims at fitting personal abilities. Ultimately, in enhancement the technology is embodied, while it remains 'other' in learning.

Different technologies have been put forward for the various types of enhancement. Cyborg practice has highlighted the importance of exoskeletons and extra limbs or sense for physical enhancement. Bostrom and Sandberg (2009) propose augmented and virtual reality shells, smart wearables (insideables, embeddables, accessories) for cognitive augmentation. Wearables and affective computing might be used to monitor and trigger individuals' emotional states.

As enhancement is directed towards psychological, cognitive, and physical abilities, designing for enhancement requires understanding of medicine, psychology, and cognitive sciences. Enhancement technologies can and should be evaluated regarding physiological measurements of the abilities and symptoms they are meant to have an impact on, so that designing for enhancement requires interdisciplinary work. The following underlines details on the mechanism considered for the design of the technology, knowing that these choices should be informed by collaboration with the disciplines just mentioned.

\subsection{Transposing enhancement to organisations}

Organisations can be seen as collective bodies which can be enhanced. We propose three strategies to achieve organisational enhancement:

(i) The organisation gives access to augmentation technology to individuals for the development of their own abilities, without relationship to work, as it has occurred in Sweden, Belgium, and the USA (Astor 2017). Here, the organisation gives the infrastructure for some kind of personal enhancement. This raises issues in terms of ownership of the technology, data collected from it, and possible harmful consequences.

(ii) The organisation gives access to augmentation technology to individuals so as to help them improve abilities in relation to their work, be it global abilities or taskrelated forms of enhancement. This raises issues in terms of consent, management, privacy, and possible harmful consequences (Gauttier, 2017). This enhancement can of physical, cognitive, or psychological nature, and improve the moral character of individuals. In turn, the organisation is enhanced as the sum of individuals composing it are enhanced as well. Organisational enhancement can be seen as an enhancement of performance, of mood (satisfaction and well-being in the organisation), of competencies (improved abilities of the organisation), through the improved abilities (cognitive, physical, psychological) of the employees. This requires that the objective of the enhancement satisfies fits with the individual and organisational endeavors. It also requires that the technology is designed so as to enhance the individual without disturbing organisational processes, or a higher readiness to transformation where the organisation realizes that the introduction of technology into staff management ultimately leads to redefining management practices, team and individual work.

(iii) The organisation uses technology in order to enhance itself. Such a scenario requires thinking of the organisation not as the sum of individuals that compose it, but as a separate entity, achieving something transcending the sum of the achievements of its individual members. In other words, it characterizes the 'organisational' part of the organisation. Enhancing the cognition of the 
organisation can be assimilated to the enhancement of the intelligence of an organisation and its decision-making processes. Enhancing an organisation physically might be possible through its infrastructure. It can raise issues in terms of transparency in decision-making, for instance when using robots with voting rights in boards of directors as a way to extend cognition.

Scenarios (i) and (ii) suppose an alignment between what individuals and the organisation see as an acceptable and desirable form of enhancement. Indeed, it requires that the individual is ready to use technology in a way that will enhance the company.

This paper focuses on scenario (ii). In the context of the hospital, it means that the organisation is enhanced when it can deliver better care (qualitative side of performance), more care (understood quantitatively as treating more patients), or giving care in a more financially sound way (quantitative side of performance). It can be can achieved when the hospital delivers new services (abilities) or is relieved from organisational tensions (mood) because individual employees have better abilities (cognitive, physical, psychological) to do their jobs.

\subsection{From the ethics of individual enhancement to the ethics of organisational enhancement}

The ethics of individual enhancement are heavily discussed. Transhumanists put forward the idea of morphological freedom (Sandberg, 2013), i.e. the right of individuals to decide to use technology to improve themselves, their bodies, their abilities, however they want. However, they posit that if a substantial number of individuals decide to enhance themselves, productivity could increase exponentially (Buchanan, 2008). They argue that the cycle of diffusion of technology can be quick enough for certain technologies. Difficulties arise as transhumanists do not think they should wait to see if enhancement technologies are safe before promoting them. More (2013b) argues in favor of a pro-actionary principle that protects technological experimentation and progress. Thinkers who are more reserved towards enhancement have argued it can alter the meaning of life (Kass, 1997) or experiences, diminish individuals' personalities and resistance to adversity (Chatterjee, 2007). It might impair justice and be a worry in terms of constraint and freedom (Chatterjee, 2007). Indeed, when more and more individuals enhance themselves, one needs to do the same to remain 'competitive', so that the idea of freedom and true consent to enhancement might not exist. There can be an issue of justice if enhancement technologies are available only for a specific population to the detriment of others.
Such issues transpose to the context of organizational enhancement. Indeed, one can question the freedom of an individual employee in accepting the enhancement technology if some other employees accept it. The purpose of enhancement in the scenario we explore in organization is not driven by morphological freedom but accomplishing tasks for a job, and the power relationship between organization and staff might contravene the idea of freedom and consent. The impact on autonomy and personal identity is reinforced. Additional ethical issues related to how the technology is handled (data ownership, use, privacy, security, safety) can be raised, in similar manners as for other technologies used at work.

Enhancing hospital and nurses therefore necessitates to take into account the ethical issues related to enhancement and address them through technology design and implementation.

\section{ENHANCING NURSES IN THE HOSPITAL}

\subsection{Nurses' enhancement needs}

Independently of their specialty, nurses are facing a series of issues identified by Chen et al (2016) as:

- Conflict with personal values, which occur when nurses have to follow instructions by doctors which are in contradiction with the patients' or family's wishes;

- Excessive workload, related to increases in administrative tasks and shortages of staff, which causes loss of concentration and a poorer quality of work ;

- Curved autonomy, referring to having to follow doctors' instructions or not being able to do anything due to inconsistent opinions;

- Constraints engendered by organisational norms such as norms allowing to move patients between wards, leading nurses to care for patients whose diseases they are not trained on, leading to poorer care and higher moral distress;

- Self-expectation frustration, related to the impossibility for nurses to advance their studies or the quality of care that they deliver as they lack time.

Nurses do not feel empowered and perceived a lack of organisational support. The lack of empowerment means nurses feel they have no control and little influence on outcomes, which leads to discouragement at work, but also feeling of being ineffective, decreased job satisfaction and higher risks of burnout, as well decision-making abilities and job performance (Al-Dweik et al., 2016). Often, it 
is resolved by including nurses into decision-making processes. Instead, enhancement focuses on their personal abilities.

Technology could help nurses to solve several of the issues mentioned above:

(i) Give them means to be less stressed, more focused, and so give care of higher quality by monitoring their fatigue and nervousness and triggering appropriate responses. This fights frustration, negative feelings associated to excessive workloads, and lack of autonomy.

(ii) Enhance their memory so as to have information about diseases they are not often confronted to, which helps them increasing their capacity to deal with organisational constraints.

Physical enhancement can be considered as well:

(iii) Relieve physical pain linked to repetitive movements and lifting or carrying patients.

Insideable wearables could be used to extend the mind by acting as extra memory where nurses could store information on treatment procedures for less common diseases, leading to a truly embodied relation to work and central place of work in the body and identity of the individual nurse. Wearables have also been mentioned in the literature as a potential tool to measure nurses' fatigue (Jovanov et al., 2011). Using wearables to monitor stress and fatigue is already possible for individual users who can use accessories such as watches or headband devices gathering data on some of the following aspects: skin conductivity, heartbeat, blood pressure, brain activity, sleep patterns, breathing patterns attention levels, voice patterns. Specific devices are also designed not only to monitor these elements but give the user elements to calm down such as music or mediation exercises. It is therefore realistic to imagine the use of such devices by nurses for them to a) become aware of when their energy and attention levels go down, or stress goes up; and b) benefit from technology to suggest appropriate steps for nurses to go back to an optimal level of energy and stress. This can be achieved through visible (external) or invisible technology (embeddable, insideable) capturing data as described above. The choice of format may have an influence onto how the technology is accepted by nurses. The feedback given can be continuous or not, and signals can be more or less disruptive. This can have an impact in nurses' self-management, with them realizing they do need a form of break or breathing exercise while there is an emergency situation or when their manager cannot afford to change the workload. It might therefore come in contradiction with the functioning of the organization. One could argue the functioning of the organization will be changed due to the transformative aspect of enhancement. Communications between nurses and team work might be affected by more individual tempo of work advocated by wearables. Exoskeletons can be used to relieve back pain, simply enhancing the comfort of nurses, so they can go through their shift and reallocate energy to other (cognitive) processes.

\subsection{Elements to consider for an ethical development of enhancing wearables for nurses}

First of all, organizational enhancement itself requires to have specific procedures allowing for true consent, with the possibility of opting out, without being penalized in any way (tasks, salary, promotion, recruitment) so as to respect the individual. It also requires guaranteeing how data will be used, stored, and protection of staff's privacy. The autonomy of the individuals should also be preserved, through technological solutions or through hospital policy. Long term impact on development (muscles with exoskeletons, emotional intelligence with other wearables) must be considered.

Introducing enhancement wearables in hospitals is bound to have an impact on patients. Firstly, it can change their perception of the nursing staff: exoskeletons can transform the image of the nursing staff to an almost nursed or disabled one, indicating that invisible technologies might be more easily accepted. It can change the way they communicate, as technology can give nurses interrupting feedback or delaying how they approach a patient. To be enhancing the hospital, the wearables must have impact on the quality of care, and so on patients' satisfaction and experience. Associating patients to the development of the technology is crucial.

\section{Conclusions}

Applying the concept of enhancement to the workplace allows to think of a new role for digital technologies. However, it is a practice that raises a series of questions linked to its ethical, social, and organisational impact. There is potential mismatch between what individuals need to be enhanced and how they are expected to behave by the organisation or stakeholders. Future research should investigate how this misalignment occurs and can be solved. The ethical and societal issues raised are intrinsically linked to the idea of enhancement (personal aspect, identity, consent), reinforced by the organisational aspect (respect of privacy and autonomy vs data gathered). However, moving the discourse on enhancement from concepts and ideologies to the design of technology and accompanying policies might allow to identify formats and conditions of acceptability and acceptance of enhancement technologies. 


\section{References}

Al-Dweik, G., Al-Daken, L. I., Abu-Snieneh, H., \& Ahmad, M. M. (2016). Work-related empowerment among nurses: literature review. International Journal of Productivity and Quality Management, 19(2), 168-186.

Anderson, R. (2012). Why cognitive enhancement is in your future (and your past). The Atlantic, 6 .

Astor, M., (2017), Microchip Implants for Employees? One Company Says Yes, NY Times

Baertschi, B. (2011), Chapitre 4. L'humanité se dit de multiples manières. Journal International de Bioéthique Vol. 22, 67-76.

Bostrom, N., Sandberg, A. (2009), Cognitive Enhancement: Methods, Ethics, Regulatory Challenges. Science and Engineering Ethics 15, 311-341.

Buchanan, A. (2008), Enhancement and the Ethics of Development. Kennedy Institute of Ethics Journal, 18, 1-34.

Chatterjee A. (2007), " Cosmetic Neurology and Cosmetic Surgery: Parallels, Predictions, and Challenges ", Cambridge Quarterly of Healthcare Ethics, no 16, p. 131.

Chen, Pei-Pei, Hsiao-Lu Lee, Shu-He Huang, Ching-Ling Wang, Chiu-Mieh Huang (in press). Nurses' perspectives on moral distress: A Q methodology approach. Nursing Ethics.

Engelbart, D.C., (1962). A Research Center for Augmenting Human Intellect, in: Proceedings of the December 9-11, 1968, Fall Joint Computer Conference, Part I, AFIPS '68 (Fall, Part I). ACM, New York, NY, USA, pp. 395-410.

Fukuyama, F. (2002), Our posthuman future : consequences of the biotechnology revolution, Farrar Straus \& Giroux

Gauttier S., (2017), Vers une 'entreprise augmentée' : de nouveaux challenges pour la recherche en Management et Systèmes d'Information, Terminal, 120

Jovanov, E., Frith, K., Anderson, F., Milosevic, M., \& Shrove, M. T. (2011, August). Real-time monitoring of occupational stress of nurses. In Engineering in Medicine and Biology Society, EMBC, 2011 Annual International Conference of the IEEE (pp. 3640-3643). IEEE.
Kass, L. (1997), The wisdom of repugnance, The new republic, retrieved from: http://web.stanford.edu/ mvr2j/sfsu09/extra/Kass 2.pdf

More, M. (2013a), 'The philosophy of Transhumanism', in The Transhumanist Reader: Classical and Contemporary Essays on the Science, Technology, and Philosophy of the Human Future, in More, M., Vita- More, N. (Eds.), 1 edition. ed. Wiley-Blackwell, Chichester, West Sussex, UK

More, M. (2013b), 'The proactionary principle: optimizing technological outcomes', in The Transhumanist Reader: Classical and Contemporary Essays on the Science, Technology, and Philosophy of the Human Future, in More, M., Vita-More, N. (Eds.), 1 edition. ed. Wiley-Blackwell, Chichester, West Sussex, UK

Sandberg, A. (2013). 'An overview of models of technological singularity', in The Transhumanist Reader: Classical and Contemporary Essays on the Science, Technology, and Philosophy of the Human Future, in More, M., Vita-More, N. (Eds.), 1 edition. ed. Wiley-Blackwell, Chichester, West Sussex, UK

Wainwright, O. (2015), Body-hackers: the people who turn themselves into cyborgs, The Guardian

Xia, C., Maes, P., 2013. The Design of Artifacts for Augmenting Intellect, in: Proceedings of the 4th Augmented Human International Conference, AH '13. ACM, New York, NY, USA, pp. 154-161. doi:10.1145/2459236.2459263

\section{Acknowledgements}

This project has received funding from the European Union's Horizon 2020 research and innovation programme under the Marie Sklodowska-Curie grant agreement No 795536. 